ICIFSS, 8-10 January 2018, Erode, Tamil Nadu, India

Notes on Intuitionistic Fuzzy Sets

Print ISSN 1310-4926, Online ISSN 2367-8283

Vol. 24, 2018, No. 1, 48-59

DOI: $10.7546 /$ nifs.2018.24.1.48-59

\title{
On the intuitionistic fuzzy polynomial ideals of a ring
}

\author{
P. K. Sharma ${ }^{1}$ and Gagandeep Kaur ${ }^{2}$ \\ ${ }^{1}$ Post Graduate Department of Mathematics, D.A.V. College \\ Jalandhar, Punjab, India \\ e-mail: pksharma@davjalandhar.com \\ ${ }^{2}$ Research Scholar, IKG PT University \\ Jalandhar, Punjab, India \\ e-mail: taktogagan@gmail.com
}

Received: 19 September 2017 Revised: 26 November 2017 Accepted: 30 November 2017

\begin{abstract}
In this paper we introduce the notion of intuitionistic fuzzy polynomial ideal $A_{x}$ of a polynomial ring $R[x]$ induced by an intuitionistic fuzzy ideal $A$ of a ring $R$, and obtain an isomorphism theorem of a ring of intuitionistic fuzzy cosets of $A_{x}$. It is shown that an intuitionistic fuzzy ideal $A$ of a ring is an intuitionistic fuzzy prime if and only if $A_{x}$ is an intuitionistic fuzzy prime ideal of $R[x]$. Moreover, we show that if $A_{x}$ is an intuitionistic fuzzy maximal ideal of $R[x]$, then $A$ is an intuitionistic fuzzy maximal ideal of $R$ but converse is not true.
\end{abstract}

Keywords: Intuitionistic fuzzy polynomial ideal, Intuitionistic fuzzy ideal, $f$-invariant, Intuitionistic fuzzy prime (maximal) ideal.

2010 Mathematics Subject Classification: 03E72, 03F55, 13 F20.

\section{Introduction}

One of the remarkable generalizations of the fuzzy sets is the intuitionistic fuzzy sets which was introduced by Atanassov [1, 2]. Biswas was the first one to introduce the intuitionistic fuzzification of the algebraic structure and developed the concept of intuitionistic fuzzy subgroup of 
a group in [5]. Later on, Hur and others in [6] and [7] defined and studied intuitionistic fuzzy subrings and ideals of a ring. With a different approach, Mukerjee and Basnet in [4] also studied intuitionistic fuzzy subrings of a ring. Jun and others in [8] introduced and study the notion of intuitionistic nil radicals of intuitionistic fuzzy ideals and Euclidean intuitionistic fuzzy ideals in rings. Translate of intuitionistic fuzzy subring and ideal was studied by Sharma in [14]. Meena and Thomas in [13] studied the concept of intuitionistic fuzzy subring to lattice setting and introduced the notion of intuitionistic L-fuzzy subring. The concept of characteristic intuitionistic fuzzy subrings of an intuitionistic fuzzy ring was introduced by Meena in [12]. In this paper, we introduce the notion of intuitionistic fuzzy polynomial ideal of a ring and study some of their properties.

\section{Preliminaries}

In this section, we review some definitions which will be used in the later section. Throughout this paper unless stated otherwise all rings are commutative rings with identity.

Definition 2.1. ([3,4]) Let $R$ be a ring. An IFS $A=\left(\mu_{A}, \nu_{A}\right)$ of $R$ is said to be an intuitionistic fuzzy ideal (IFI) of $R$ if

(i) $\mu_{A}(x-y) \geq \mu_{A}(x) \wedge \mu_{A}(y)$ and $\nu_{A}(x-y) \leq \nu_{A}(x) \vee \nu_{A}(y)$;

(ii) $\mu_{A}(x y) \geq \mu_{A}(x) \vee \mu_{A}(y)$ and $\nu_{A}(x y) \leq \nu_{A}(x) \wedge \nu_{A}(y), \forall x, y \in R$.

Definition 2.2. ([8]) Let $R$ and $S$ be any sets and let $f: R \rightarrow S$ be a function. An IFS $A$ of $R$ is called an $f$-invariant if $f(x)=f(y) \Rightarrow \mu_{A}(x)=\mu_{A}(y)$ and $\nu_{A}(x)=\nu_{A}(y)$, where $x, y \in R$.

If $A$ is any $f$-invariant IFS of $R$, then $f^{-1}(f(A))=A$.

The following results are easy to prove:

Lemma 2.3. Let $R$ and $S$ be any sets and $f: R \rightarrow S$ be any function. If $A$ and $B$ are IFS of $R$ and $S$ respectively are $f$-invariant, then $A \cup B$ and $A \cap B$ are $f$-invariant.

Lemma 2.4. Let $R$ and $S$ be any sets and $f: R \rightarrow S$ be any function. Let $A$ and $B$ be $f$-invariant IFS of $R$. If $A \subseteq B$, then $f(A) \subseteq f(B)$.

Theorem 2.5. Let $f: R \rightarrow R^{\prime}$ be a homomorphism of rings. If $A$ and $B$ are $f$-invariant IFS of $R$ and $R^{\prime}$ respectively. Then

(i) $(f(A))_{*}=f\left(A_{*}\right)$

(ii) $\left(f^{-1}(B)\right)_{*}=f^{-1}\left(B_{*}\right)$.

Let $R$ be a commutative ring with identity and let $R[x]$ be the ring of polynomials where $x$ is an indeterminate.

Definition 2.6. Let $f: R \rightarrow R^{\prime}$ be a homomorphism of rings. A map $f_{x}: R[x] \rightarrow R^{\prime}[x]$ defined by

$$
f_{x}\left(\sum_{i=0}^{n} a_{i} x^{i}\right)=\sum_{i=0}^{n} f\left(a_{i}\right) x^{i},
$$

is obviously a ring homomorphism, and we call $f_{x}$ an induced homomorphism by $f$. 


\section{Intuitionistic fuzzy polynomial ideals}

In this section, we introduce the notion of intuitionistic fuzzy polynomial ideal of a ring and study their properties. The set of all real numbers is denoted by $\mathbf{R}$.

Lemma 3.1. Let $a_{i}, b_{i} \in \boldsymbol{R}(i=1,2, \ldots, n)$. Then

$\min _{i}\left[\min \left\{a_{i}, b_{i}\right\}\right]=\min \left\{\min _{i}\left(a_{i}\right), \min _{i}\left(b_{i}\right)\right\}$ and $\max _{i}\left[\max \left\{a_{i}, b_{i}\right\}\right]=\max \left\{\max _{i}\left(a_{i}\right), \max _{i}\left(b_{i}\right)\right\}$

Proof. Straightforward.

Lemma 3.2. Let $a_{i}, b_{i} \in \boldsymbol{R}(i=1,2, \ldots, n)$. Then

$\min _{i}\left[\max \left\{a_{i}, b_{i}\right\}\right] \geq \max \left\{\min _{i}\left(a_{i}\right), \min _{i}\left(b_{i}\right)\right\}$ and $\max _{i}\left[\min \left\{a_{i}, b_{i}\right\}\right] \leq \min \left\{\max _{i}\left(a_{i}\right), \max _{i}\left(b_{i}\right)\right\}$

Proof. Straightforward.

Lemma 3.3. Let $A=\left(\mu_{A}, \nu_{A}\right)$ be an IFI of a ring $\boldsymbol{R}$. Then

$\mu_{A}\left(a_{1} b_{n}+a_{2} b_{n-1}+\cdots+a_{n} b_{1}\right) \geq \max \left\{\min _{i}\left(\mu_{A}\left(a_{i}\right)\right), \min _{i}\left\{\mu_{A}\left(b_{i}\right)\right\}\right\}$ and

$\nu_{A}\left(a_{1} b_{n}+a_{2} b_{n-1}+\cdots+a_{n} b_{1}\right) \leq \min \left\{\max _{i}\left(\nu_{A}\left(a_{i}\right)\right), \max _{i}\left\{\nu_{A}\left(b_{i}\right)\right\}\right\}, \forall a_{i}, b_{i} \in \boldsymbol{R}$.

Proof. Since $A=\left(\mu_{A}, \nu_{A}\right)$ be an IFI of a ring $\mathbf{R}$. for any $a_{i}, b_{i} \in \mathbf{R}(i=1,2, \ldots, n)$.

By Definition (2.1), we have

$$
\begin{aligned}
\mu_{A}\left(a_{1} b_{n}+a_{2} b_{n-1}+\cdots+a_{n} b_{1}\right) & \geq \min _{i}\left\{\mu_{A}\left\{a_{i} b_{n+1-i}\right\}\right\} \\
& \geq \min _{i}\left\{\max \left\{\mu_{A}\left(a_{i}\right), \mu_{A}\left(b_{n+1-i}\right)\right\}\right\} \\
& \geq \max _{i}\left\{\min _{i}\left\{\mu_{A}\left(a_{i}\right), \mu_{A}\left(b_{i}\right)\right\}\right\} \text { and } \\
\nu_{A}\left(a_{1} b_{n}+a_{2} b_{n-1}+\cdots+a_{n} b_{1}\right) & \leq \max _{i}\left\{\nu_{A}\left\{a_{i} b_{n+1-i}\right\}\right\} \\
& \leq \max _{i}\left\{\min \left\{\nu_{A}\left(a_{i}\right), \nu_{A}\left(b_{n+1-i}\right)\right\}\right\} \\
& \leq \min _{i}\left\{\max _{i}\left\{\nu_{A}\left(a_{i}\right), \nu_{A}\left(b_{i}\right)\right\}\right\} .
\end{aligned}
$$

Theorem 3.4. Let $A=\left(\mu_{A}, \nu_{A}\right)$ be an IFI of a ring $R$ and let $f(x)=\sum_{i=0}^{n} a_{i} x^{i} \in R[x]$.

Define an IFS $A_{x}=\left(\mu_{A_{x}}, \nu_{A_{x}}\right)$ on $R[x]$ by

$$
\mu_{A_{x}}(f(x))=\min _{i}\left\{\mu_{A}\left(a_{i}\right)\right\} \text { and } \nu_{A_{x}}(f(x))=\max _{i}\left\{\nu_{A}\left(a_{i}\right)\right\} .
$$

Then $A_{x}$ is an IFI of $R[x]$.

Proof. By Definition (2.1), we show that $A_{x}$ is an IFI of $R[x]$.

Let $f(x)=\sum_{i=0}^{n} a_{i} x^{i}$ and $g(x)=\sum_{i=0}^{n} b_{i} x^{i} \in R[x]$. Then by Lemma (3.1), we have

$$
\begin{aligned}
\mu_{A_{x}}(f(x)-g(x)) & =\min _{i}\left\{\mu_{A}\left(c_{i}\right)\right\}, \text { where } c_{i}=a_{i}-b_{i} \\
& =\min _{i}\left\{\mu_{A}\left(a_{i}-b_{i}\right)\right\} \\
& \geq \min _{i}\left\{\min \left\{\mu_{A}\left(a_{i}\right), \mu_{A}\left(b_{i}\right)\right\}\right\} \\
& =\min \left\{\min _{i}\left\{\mu_{A}\left(a_{i}\right)\right\}, \min _{i}\left\{\mu_{A}\left(b_{i}\right)\right\}\right\} \\
& =\min \left\{\mu_{A_{x}}(f(x)), \mu_{A_{x}}(g(x))\right\} .
\end{aligned}
$$


Thus, $\mu_{A_{x}}(f(x)-g(x)) \geq \min \left\{\mu_{A_{x}}(f(x)), \mu_{A_{x}}(g(x))\right\}$. Similarly, we can show that $\nu_{A_{x}}(f(x)-g(x)) \leq \max \left\{\nu_{A_{x}}(f(x)), \nu_{A_{x}}(g(x))\right\}$. Also,

$$
\begin{aligned}
\mu_{A_{x}}(f(x) g(x)) & =\min _{i}\left\{\mu_{A}\left(d_{i}\right)\right\}, \text { where } d_{i}=\sum_{i}^{n+m} a_{i} b_{n+m-i} \\
& =\min _{i}\left\{\max \left\{\mu_{A}\left(a_{i}\right)\right\},\left\{\mu_{A}\left(b_{n+m-i}\right)\right\}\right\} \\
& \geq \min _{i}\left\{\max \left\{\mu_{A}\left(a_{i}\right), \mu_{A}\left(b_{i}\right)\right\}\right\} \\
& \geq \max \left\{\min _{i}\left\{\mu_{A}\left(a_{i}\right)\right\}, \min _{i}\left\{\mu_{A}\left(b_{i}\right)\right\}\right\} \\
& =\max \left\{\mu_{A_{x}}(f(x)), \mu_{A_{x}}(g(x))\right\}
\end{aligned}
$$

Thus, $\mu_{A_{x}}(f(x) g(x)) \geq \max \left\{\mu_{A_{x}}(f(x)), \mu_{A_{x}}(g(x))\right\}$. Similarly, we can show that $\nu_{A_{x}}(f(x) g(x)) \leq \min \left\{\nu_{A_{x}}(f(x)), \nu_{A_{x}}(g(x))\right\}$. This proves that $A_{x}$ is an IFI of $R[x]$.

Definition 3.5. The intuitionistic fuzzy ideal $A_{x}$ discussed in Theorem (3.4) is called the intuitionistic fuzzy polynomial ideal (IFPI) of $R[x]$ induced by an intuitionistic fuzzy ideal $A$.

Proposition 3.6. Let $f: R \rightarrow R^{\prime}$ be a homomorphism of rings and let $f_{x}: R[x] \rightarrow R^{\prime}[x]$ be an induced homomorphism of $f$. If $A$ is an IFI of the ring $R$ and $A_{x}$ be its IFPI of $R[x]$, then $A$ is $f$-invariant if and only if $A_{x}$ is $f_{x}$-invariant.

Proof. Assume that A is $f$-invariant. Let $f_{x}(r(x))=f_{x}(s(x))$, where $r(x)=\sum_{i=0}^{m} a_{i} x^{i}$ and $s(x)=\sum_{i=0}^{m} b_{i} x^{i} \in R[x]$. Then $\sum_{i=0}^{m} f\left(a_{i}\right) x^{i}=\sum_{i=0}^{m} f\left(b_{i}\right) x^{i} \Rightarrow f\left(a_{i}\right)=f\left(b_{i}\right), \forall i=1,2, \ldots, m$. Hence $\mu_{A_{x}}(r(x))=\min _{i}\left\{\mu_{A}\left(a_{i}\right)\right\}=\min _{i}\left\{\mu_{A}\left(b_{i}\right)\right\}=\mu_{A_{x}}(s(x))$ and $\nu_{A_{x}}(r(x))=\max _{i}\left\{\nu_{A}\left(a_{i}\right)\right\}$ $=\max _{i}\left\{\nu_{A}\left(b_{i}\right)\right\}=\nu_{A_{x}}(s(x))$. Thus, $A_{x}$ is $f_{x}$-invariant.

Conversely, assume that $A_{x}$ is an $f_{x}$-invariant. If $f(a)=f(b)$, then $f_{x}(a)=f_{x}(b)$. Since $A_{x}$ is an $f_{x}$-invariant. So, we have $\mu_{A_{x}}(a)=\mu_{A_{x}}(b)$ and $\nu_{A_{x}}(a)=\nu_{A_{x}}(b)$, which implies that $\mu_{A}(a)=\mu_{A}(b)$ and $\nu_{A}(a)=\nu_{A}(b)$. Thus $A$ is $f$-invariant.

Proposition 3.7. Let $A$ be an IFI of the ring $R$. Then the set

$$
S=\left\{f(x) \in R[x]: \mu_{A_{x}}(f(x))=\mu_{A_{x}}(0) \text { and } \nu_{A_{x}}(f(x))=\nu_{A_{x}}(0)\right\}
$$

is a subring of $R[x]$.

Proof. Let $f(x), g(x)$ be any two element of $S$, then

$$
\mu_{A_{x}}(f(x)-g(x)) \geq \min \left\{\mu_{A_{x}}(f(x)), \mu_{A_{x}}(g(x))\right\}=\mu_{A_{x}}(0)
$$

and

$$
\mu_{A_{x}}(f(x) g(x)) \geq \max \left\{\mu_{A_{x}}(f(x)), \mu_{A_{x}}(g(x))\right\}=\mu_{A_{x}}(0) .
$$

Similarly, we can show that $\nu_{A_{x}}(f(x)-g(x)) \leq \max \left\{\nu_{A_{x}}(f(x)), \nu_{A_{x}}(g(x))\right\}=\nu_{A_{x}}(0)$ and $\nu_{A_{x}}(f(x) g(x)) \leq \min \left\{\nu_{A_{x}}(f(x)), \nu_{A_{x}}(g(x))\right\}=\nu_{A_{x}}(0)$.

On the other hand, $\mu_{A_{x}}(f(x)) \leq \mu_{A_{x}}(0)$ and $\nu_{A_{x}}(f(x)) \geq \nu_{A_{x}}(0), \forall f(x) \in R[x]$.

So, $f(x)-g(x), f(x) g(x) \in S$. Thus, $S$ is a subring of $R[x]$. 
Remark 3.8. Let $A$ be an IFS of a ring $R$. We denote a level cut set $A_{*}$ by

$$
A_{*}=\left\{x \in R: \mu_{A}(x)=\mu_{A}(0) \text { and } \nu_{A}(x)=\nu_{A}(0)\right\} .
$$

It is proved in [11] that if $A$ is an IFI of ring $R$, then $A_{*}$ is an ideal of ring $R$. Note that if $A$ is an IFI of a ring $R$, then $\mu_{A}(0) \geq \mu_{A}(x)$ and $\nu_{A}(0) \leq \nu_{A}(x)$ for all $x \in R$.

We denote $A_{*}[x]=\left\{f(x)=\sum_{i=0}^{n} a_{i} x^{i} \in R[x]\right.$ : where $\left.a_{i} \in A_{*}, \forall i=1,2, \ldots, n\right\}$.

Theorem 3.9. Let $A$ be an IFI of a ring $R$, then $\left(A_{x}\right)_{*}=A_{*}[x]$.

Proof. It follows that

$$
\begin{aligned}
\left(A_{x}\right)_{*} & =\left\{f(x) \in R[x]: f(x)=\sum_{i=0}^{n} a_{i} x^{i}, \mu_{A_{x}}(f(x))=\mu_{A_{x}}(0) \text { and } \nu_{A_{x}}(f(x))=\nu_{A_{x}}(0)\right\} \\
& =\left\{f(x) \in R[x]: f(x)=\sum_{i=0}^{n} a_{i} x^{i}, \min _{i}\left\{\mu_{A}\left(a_{i}\right)\right\}=\mu_{A}(0) \text { and } \nu_{A}\left(a_{i}\right)=\nu_{A}(0)\right\} \\
& =\left\{f(x) \in R[x]: f(x)=\sum_{i=0}^{n} a_{i} x^{i}, \mu_{A}\left(a_{i}\right)=\mu_{A}(0) \text { and } \nu_{A}\left(a_{i}\right)=\nu_{A}(0), \forall i\right\} \\
& =\left\{f(x) \in R[x]: f(x)=\sum_{i=0}^{n} a_{i} x^{i}, a_{i} \in A_{*}, \forall i\right\}=A_{*}[x] .
\end{aligned}
$$

Theorem 3.10. If $A$ and $B$ are two IFIs of a ring $R$, then

(i) $(A \cap B)_{x}=A_{x} \cap B_{x}$.

(ii) $(A \cup B)_{x} \supseteq A_{x} \cup B_{x}$.

(iii) $A_{x}+B_{x} \subseteq(A+B)_{x}$.

(iv) $A_{x} B_{x} \subseteq(A B)_{x}$.

Proof. Let $f(x)=\sum_{i=0}^{n} a_{i} x^{i}$ be any element of $R[x]$, then

(i) $(A \cap B)_{x}(f(x))=\left(\mu_{(A \cap B)_{x}}(f(x)), \nu_{(A \cap B)_{x}}(f(x))\right)$, where

$$
\begin{aligned}
\mu_{(A \cap B)_{x}} & =\min _{i}\left\{\mu_{(A \cap B)}\left(a_{i}\right)\right\} \\
& =\min _{i}\left\{\min \left\{\mu_{A}\left(a_{i}\right), \mu_{B}\left(a_{i}\right)\right\}\right\} \\
& =\min _{i}\left\{\min _{i}\left\{\mu_{A}\left(a_{i}\right), \mu_{B}\left(a_{i}\right)\right\}\right\}[\text { Using Lemma (3.1)] } \\
& =\min _{i}\left\{\min _{i}\left\{\mu_{A}\left(a_{i}\right)\right\}, \min _{i}\left\{\mu_{B}\left(a_{i}\right)\right\}\right\} \\
& =\min \left\{\mu_{A_{x}}(f(x)), \mu_{B_{x}}(f(x))\right\} \\
& =\mu_{A_{x} \cap B_{x}}(f(x)) .
\end{aligned}
$$

Similarly, we can show that $\nu_{(A \cap B)_{x}}=\nu_{A_{x} \cap B_{x}}(f(x))$. Hence $(A \cap B)_{x}=A_{x} \cap B_{x}$. (ii) $(A \cup B)_{x}(f(x))=\left(\mu_{(A \cup B)_{x}}(f(x)), \nu_{(A \cup B)_{x}}(f(x))\right)$, where

$$
\begin{aligned}
\mu_{(A \cup B)_{x}} & =\min _{i}\left\{\mu_{(A \cup B)}\left(a_{i}\right)\right\} \\
& =\min _{i}\left\{\max \left\{\mu_{A}\left(a_{i}\right), \mu_{B}\left(a_{i}\right)\right\}\right\} \\
& \geq \max \left\{\min _{i}\left\{\mu_{A}\left(a_{i}\right), \mu_{B}\left(a_{i}\right)\right\}\right\} \text { Using Lemma (3.2)] } \\
& =\max \left\{\min _{i}\left\{\mu_{A}\left(a_{i}\right)\right\}, \min _{i}\left\{\mu_{B}\left(a_{i}\right)\right\}\right\} \\
& =\max \left\{\mu_{A_{x}}(f(x)), \mu_{B_{x}}(f(x))\right\} \\
& =\mu_{A_{x} \cup B_{x}}(f(x)) .
\end{aligned}
$$


Similarly, we can show that $\nu_{(A \cup B)_{x}} \leq \nu_{A_{x} \cup B_{x}}(f(x))$. Hence $(A \cup B)_{x} \supseteq A_{x} \cup B_{x}$.

(iii) Now, $\left(A_{x}+B_{x}\right)(f(x))=\left(\mu_{A_{x}+B_{x}}(f(x)), \nu_{A_{x}+B_{x}}(f(x))\right)$, where

$$
\begin{aligned}
\mu_{A_{x}+B_{x}}(f(x)) & =\max _{f(x)=g(x)+h(x)}\left\{\min \left\{\mu_{A_{x}}(g(x)), \mu_{B_{x}}(h(x))\right\}\right\}, g(x)=\sum_{i=0}^{p} b_{i} x^{i}, h(x)=\Sigma_{i=0}^{p} c_{i} x^{i} \\
& =\max _{f(x)=g(x)+h(x)}\left\{\min \left\{\min _{i}\left\{\mu_{A}\left(b_{i}\right)\right\}, \min _{i}\left\{\mu_{B}\left(c_{i}\right)\right\}\right\}\right\} \\
& =\max _{a_{i}=b_{i}+c_{i}}\left\{\min _{i}\left\{\min \left\{\mu_{A}\left(b_{i}\right), \mu_{B}\left(c_{i}\right)\right\}\right\}\right\}[\text { Using Lemma (3.1)] } \\
& \leq \min _{i}\left\{\max _{a_{i}=b_{i}+c_{i}}\left\{\min \left\{\mu_{A}\left(b_{i}\right), \mu_{B}\left(c_{i}\right)\right\}\right\}\right\}[\text { Using Lemma (3.1)] } \\
& =\min _{i}\left\{\mu_{A+B}\left(a_{i}\right)\right\} \\
& =\mu_{(A+B)_{x}}(f(x)) .
\end{aligned}
$$

Thus, we get $\mu_{A_{x}+B_{x}}(f(x)) \leq \mu_{(A+B)_{x}}(f(x))$. Similarly, we can show that $\nu_{A_{x}+B_{x}}(f(x)) \geq \nu_{(A+B)_{x}}(f(x))$. Hence $A_{x}+B_{x} \subseteq(A+B)_{x}$.

(iv) Now, $\left(A_{x} B_{x}\right)(f(x))=\left(\mu_{A_{x} B_{x}}(f(x)), \nu_{A_{x} B_{x}}(f(x))\right)$, where

$$
\begin{aligned}
\mu_{A_{x} B_{x}}(f(x)) & =\operatorname{Sup}_{f(x)=g(x) h(x)}\left\{\min \left\{\mu_{A_{x}}(g(x)), \mu_{B_{x}}(h(x))\right\}\right\}, g(x)=\sum_{i=0}^{n} b_{i} x^{i}, h(x)=\sum_{i=0}^{m} c_{i} x^{i}, n+m=p \\
& =\operatorname{Sup}_{a_{i}=\sum_{i=0}^{n+m=p}\left(b_{i} c_{n+m-i}\right)}\left\{\min \left\{\min _{i}\left\{\mu_{A}\left(b_{i}\right)\right\}, \min _{i}\left\{\mu_{B}\left(c_{n+m-i}\right)\right\}\right\}\right\} \\
& =\operatorname{Sup}_{a_{i}=\sum_{i=0}^{n+m=p}\left(b_{i} c_{n+m-i}\right)}\left\{\min _{i}\left\{\min \left\{\mu_{A}\left(b_{i}\right), \mu_{B}\left(c_{n+m-i}\right)\right\}\right\}\right\} \text { [Using Lemma (3.1)] } \\
& \leq \min _{i}\left\{\operatorname{Sup}_{a_{i}=\sum_{i=0}^{n+m=p}\left(b_{i} c_{n+m-i}\right)}\left\{\min \left\{\mu_{A}\left(b_{i}\right), \mu_{B}\left(c_{n+m-i}\right)\right\}\right\}\right\} \text { [Using Lemma (3.2)] } \\
& =\min _{i}\left\{\mu_{A B}\left(a_{i}\right)\right\} \\
& =\mu_{(A B)_{x}}(f(x)) .
\end{aligned}
$$

Thus, we get $\mu_{A_{x} B_{x}}(f(x)) \leq \mu_{(A B)_{x}}(f(x))$. Similarly, we can show that $\nu_{A_{x} B_{x}}(f(x)) \geq \nu_{(A B)_{x}}(f(x))$. Hence $A_{x} B_{x} \subseteq(A B)_{x}$.

Theorem 3.11. Let $f: R \rightarrow R^{\prime}$ be a homomorphism from $R$ onto $R^{\prime}$. If $A$ and $B$ are IFIs of $R^{\prime}$, then

(i) $f^{-1}(A \cap B)=f^{-1}(A) \cap f^{-1}(B)$

(ii) $f^{-1}(A \cup B)=f^{-1}(A) \cup f^{-1}(B)$.

Proof. Let $x \in R$ be any element.

(i) Now, $f^{-1}(A \cap B)(x)=\left(\mu_{f^{-1}(A \cap B)}(x), \nu_{f^{-1}(A \cap B)}(x)\right)$, where

$$
\begin{aligned}
\mu_{f^{-1}(A \cap B)}(x) & =\mu_{(A \cap B)}(f(x)) \\
& =\min \left\{\mu_{A}(f(x)), \mu_{B}(f(x))\right\} \\
& =\min \left\{\mu_{f^{-1}(A)}(x), \mu_{f^{-1}(B)}(x)\right\} \\
& =\mu_{f^{-1}(A) \cap f^{-1}(B)}(x) .
\end{aligned}
$$

Similarly, we can show that $\nu_{f^{-1}(A \cap B)}(x)=\nu_{f^{-1}(A) \cap f^{-1}(B)}(x)$.

Hence $f^{-1}(A \cap B)=f^{-1}(A) \cap f^{-1}(B)$. 
(ii) Now, $f^{-1}(A \cup B)(x)=\left(\mu_{f^{-1}(A \cup B)}(x), \nu_{f^{-1}(A \cup B)}(x)\right)$, where

$$
\begin{aligned}
\mu_{f^{-1}(A \cup B)}(x) & =\mu_{(A \cup B)}(f(x)) \\
& =\max \left\{\mu_{A}(f(x)), \mu_{B}(f(x))\right\} \\
& =\max \left\{\mu_{f^{-1}(A)}(x), \mu_{f^{-1}(B)}(x)\right\} \\
& =\mu_{f^{-1}(A) \cup f^{-1}(B)}(x) .
\end{aligned}
$$

Similarly, we can show that $\nu_{f^{-1}(A \cup B)}(x)=\nu_{f^{-1}(A) \cup f^{-1}(B)}(x)$.

Hence $f^{-1}(A \cup B)=f^{-1}(A) \cup f^{-1}(B)$.

Corollary 3.12. Let $f: R \rightarrow R^{\prime}$ be a homomorphism from $R$ onto $R^{\prime}$. Let $f_{x}$ be an induced homomorphism of $f$. If $A$ and $B$ are IFIs of $R^{\prime}$, then

(i) $f_{x}^{-1}\left((A \cap B)_{x}\right)=f_{x}^{-1}\left(A_{x}\right) \cap f_{x}^{-1}\left(B_{x}\right)$

(ii) $f_{x}^{-1}\left((A \cup B)_{x}\right)=f_{x}^{-1}\left(A_{x}\right) \cup f_{x}^{-1}\left(B_{x}\right)$.

Proof. (i) It follows from Theorem (3.10)(i) and Theorem (3.11)(ii) that $f_{x}^{-1}\left((A \cap B)_{x}\right)=f_{x}^{-1}\left(A_{x} \cap B_{x}\right)=f_{x}^{-1}\left(A_{x}\right) \cap f_{x}^{-1}\left(B_{x}\right)$.

(ii) By Theorem (3.10)(ii), we have $A_{x} \cup B_{x} \subseteq(A \cup B)_{x} \Rightarrow f_{x}^{-1}\left((A \cap B)_{x}\right) \subseteq f_{x}^{-1}\left((A \cup B)_{x}\right)$. By applying Theorem (3.10)(ii) and Theorem (3.11)(ii), we obtain $f_{x}^{-1}\left(A_{x}\right) \cap f_{x}^{-1}\left(B_{x}\right)=f_{x}^{-1}\left(A_{x} \cup B_{x}\right) \subseteq f_{x}^{-1}\left((A \cup B)_{x}\right)$, which proves (ii).

Theorem 3.13. Let $f: R \rightarrow R^{\prime}$ be a homomorphism from $R$ onto $R^{\prime}$. Let $f_{x}$ be an induced homomorphism of $f$. If $A$ is an IFI of $R^{\prime}$, then $\left(f^{-1}(A)\right)_{x}=f_{x}^{-1}\left(A_{x}\right)$.

Proof. Let $r(x)=\sum_{i=0}^{n} a_{i} x^{i}$ be any element of $R[x]$, then we have Now, $\left(f^{-1}(A)\right)_{x}(r(x))=\left(\mu_{\left(f^{-1}(A)\right)_{x}}(r(x)), \nu_{\left(f^{-1}(A)\right)_{x}}(r(x))\right)$, where

$$
\mu_{\left(f^{-1}(A)\right)_{x}}(r(x))=\min _{i}\left\{\mu_{f^{-1}(A)}\left(a_{i}\right)\right\}=\min _{i}\left\{\mu_{A}\left(f\left(a_{i}\right)\right)\right\}=\mu_{A_{x}}\left(f_{x}(r(x))\right)=\mu_{f_{x}^{-1}\left(A_{x}\right)}(r(x)) .
$$

Similarly, we can show that $\nu_{\left(f^{-1}(A)\right)_{x}}(r(x))=\nu_{f_{x}^{-1}\left(A_{x}\right)}(r(x))$.

Hence $\left(f^{-1}(A)\right)_{x}=f_{x}^{-1}\left(A_{x}\right)$.

Theorem 3.14. Let $f: R \rightarrow R^{\prime}$ be a homomorphism from $R$ onto $R^{\prime}$ and let $f_{x}$ be an induced homomorphism of $f$. If $A$ is an $f$-invariant IFIs of $R^{\prime}$, then $(f(A))_{x}=f_{x}\left(A_{x}\right)$.

Proof. For any polynomial $s(x):=\sum_{i=0}^{m} b_{i} x^{i} \in R[x]$, we let $h_{j}(x):=\sum_{i=0}^{m} a_{j i} x^{i} \in R[x]$. Then $A_{x}\left(h_{j}(x)\right)=\left(\mu_{A_{x}}\left(h_{j}(x)\right), \nu_{A_{x}}\left(h_{j}(x)\right)\right)$, where $\mu_{A_{x}}\left(h_{j}(x)\right)=\min _{i}\left\{\mu_{A}\left(a_{j i}\right)\right\}$ and $\nu_{A_{x}}\left(h_{j}(x)\right)=$ $\min _{i}\left\{\nu_{A}\left(a_{j i}\right)\right\}$. Assume that $f_{x}\left(h_{j}(x)\right)=s(x)$ and $f_{x}\left(h_{k}(x)\right)=s(x)$. Then $\sum_{i=0}^{m}\left(f\left(a_{j i}\right) x^{i}=\right.$ $\sum_{i=0}^{m} b_{i} x^{i}$ and $\sum_{i=0}^{m}\left(f\left(a_{k i}\right) x^{i}=\sum_{i=0}^{m} b_{i} x^{i}\right.$. It follows that $f\left(a_{j i}\right)=b_{i}=f\left(a_{k i}\right), \forall i=1,2, \ldots, m$.

Hence $\mu_{A_{x}}\left(h_{j}(x)\right)=\min _{i}\left\{\mu_{A}\left(a_{j i}\right)\right\}=\min _{i}\left\{\mu_{A}\left(a_{k i}\right)\right\}=\mu_{A_{x}}\left(h_{k}(x)\right)$. Similarly, we can show that $\nu_{A_{x}}\left(h_{j}(x)\right)=\nu_{A_{x}}\left(h_{k}(x)\right)$.

Now, $\left[f_{x}\left(A_{x}\right)\right](s(x))=\left(\mu_{f_{x}\left(A_{x}\right)}(s(x)), \nu_{f_{x}\left(A_{x}\right)}(s(x))\right)$, where

$$
\begin{aligned}
\mu_{f_{x}\left(A_{x}\right)}(s(x)) & =\operatorname{Sup}\left\{\mu_{A_{x}}\left(h_{j}(x)\right): h_{j}(x)=\Sigma_{i=0}^{m} a_{j i} x^{i} \text { such that } f_{x}\left(h_{j}(x)\right)=s(x)\right\} \\
& =\operatorname{Sup}\left\{\min _{i}\left\{\mu_{A}\left(a_{j i}\right)\right\}, j=1,2, \ldots\right\} \\
& =\mu_{A_{x}}\left(h_{j}(x)\right) .
\end{aligned}
$$


Similarly, we can show that $\nu_{f_{x}\left(A_{x}\right)}(s(x))=\nu_{A_{x}}\left(h_{j}(x)\right)$.

Now, for $i=1,2, \ldots, m$. As $A$ is $f$-invariant, we have

$$
(f(A))\left(b_{i}\right)=\left(\mu_{f(A)}\left(b_{i}\right), \nu_{f(A)}\left(b_{i}\right)\right), \text { where }
$$

$\mu_{f(A)}\left(b_{i}\right)=\operatorname{Sup}\left\{\mu_{A}\left(a_{j i}\right), a_{j i} \in R, f\left(a_{j i}\right)=b_{i}\right\}=\mu_{A}\left(a_{0 i}\right)=\mu_{A}\left(a_{1 i}\right)=\cdots=\mu_{A}\left(a_{j i}\right)$.

Similarly, we have $\nu_{f(A)}\left(b_{i}\right)=\nu_{A}\left(a_{0 i}\right)=\nu_{A}\left(a_{1 i}\right)=\cdots=\nu_{A}\left(a_{j i}\right)$. It follows from Theorem (3.4) that

$$
\begin{aligned}
\mu_{(f(A))_{x}}(s(x)) & =\min _{i}\left\{\mu_{f(A)}\left(b_{i}\right)\right\} \\
& =\min _{i}\left\{\mu_{f(A)}\left(b_{0}\right), \mu_{f(A)}\left(b_{1}\right), \ldots\right\} \\
& =\min _{i}\left\{\mu_{A}\left(a_{j 0}\right), \mu_{A}\left(a_{j 1}\right), \ldots\right\} \\
& =\mu_{A_{x}}\left\{\sum_{i=0}^{m} a_{j i}\right\} \\
& =\mu_{f_{x}\left(A_{x}\right)}(s(x)) .
\end{aligned}
$$

Similarly, we can show that $\nu_{(f(A))_{x}}(s(x))=\nu_{f_{x}\left(A_{x}\right)}(s(x))$. Hence $(f(A))_{x}=f_{x}\left(A_{x}\right)$.

Definition 3.15. Let $A$ be an IFI of a ring $R$ and let $A_{x}$ be an intuitionistic fuzzy polynomial ideal of $R[x]$. For any $f(x) \in R[x]$, define an IFS $\left(f(x)+A_{x}\right)$ on $R[x]$ by

$$
\left(f(x)+A_{x}\right)(g(x))=\left(\mu_{f(x)+A_{x}}(g(x)), \nu_{f(x)+A_{x}}(g(x))\right), \text { where }
$$

$\mu_{f(x)+A_{x}}(g(x))=\mu_{A_{x}}(f(x)-g(x))$ and $\nu_{f(x)+A_{x}}(g(x))=\nu_{A_{x}}(f(x)-g(x)), \forall f(x), g(x) \in R[x]$. Then $f(x)+A_{x}$ is called an intuitionistic fuzzy coset of $R[x]$ determined by $f(x)$ and $A_{x}$.

Theorem 3.16. Let $A$ be an IFI of a ring $R$ and let $A_{x}$ be an intuitionistic fuzzy polynomial ideal of $R[x]$. Then $R[x] / A_{x}$, the set of all intuitionistic fuzzy cosets of $A_{x}$ form a ring under the composition defined by

$$
\begin{gathered}
\left(f(x)+A_{x}\right)+\left(g(x)+A_{x}\right):=(f(x)+g(x))+A_{x} \text { and } \\
\left(f(x)+A_{x}\right)\left(g(x)+A_{x}\right):=(f(x) g(x))+A_{x}, \forall f(x), g(x) \in R[x] .
\end{gathered}
$$

Proof. Straightforward result.

Lemma 3.17. Let $A$ be an IFI of $a$ ring $R$ and let $A_{x}$ be an intuitionistic fuzzy polynomial ideal of $R[x]$. Then $f(x)+A_{x}=g(x)+A_{x}$ if and only if $A_{x}(f(x)-g(x))=A_{x}(0)$, for all $f(x), g(x) \in R[x]$.

Proof. Firstly, assume that $f(x)+A_{x}=g(x)+A_{x}$. Then $\left(f(x)+A_{x}\right)(f(x))=\left(g(x)+A_{x}\right)(f(x))$ implies that $\left(\mu_{A_{x}}(f(x)-f(x)), \nu_{A_{x}}(f(x)-f(x))\right)=\left(\mu_{A_{x}}(g(x)-f(x)), \nu_{A_{x}}(g(x)-f(x))\right)$ i.e., $\left(\mu_{A_{x}}(0), \nu_{A_{x}}(0)\right)=\left(\mu_{A_{x}}(g(x)-f(x)), \nu_{A_{x}}(g(x)-f(x))\right)$

$\Rightarrow \mu_{A_{x}}(g(x)-f(x))=\mu_{A_{x}}(0)$ and $\left.\left.\nu_{A_{x}}(g(x)-f(x))\right)=\nu_{A_{x}}(0)\right)$

$\Rightarrow A_{x}(g(x)-f(x))=A_{x}(0)$.

Conversely, assume that $A_{x}(g(x)-f(x))=A_{x}(0)$, for all $f(x), g(x) \in R[x]$.

Consider $h(x) \in R[x]$ be any element, then we have

$$
\left(f(x)+A_{x}\right)(h(x))=\left(\mu_{f(x)+A_{x}}(h(x)), \nu_{f(x)+A_{x}}(h(x))\right),
$$


where

$$
\begin{aligned}
\mu_{f(x)+A_{x}}(h(x)) & =\mu_{A_{x}}(h(x)-f(x)) \\
& =\mu_{A_{x}}(h(x)-g(x)+g(x)-f(x)) \\
& \geq \min \left\{\mu_{A_{x}}(h(x)-g(x)), \mu_{A_{x}}(g(x)-f(x))\right\} \\
& =\min \left\{\mu_{A_{x}}(h(x)-g(x)), \mu_{A_{x}}(0)\right\} \\
& =\mu_{A_{x}}(h(x)-g(x)) \\
& =\mu_{g(x)+A_{x}}(h(x)) .
\end{aligned}
$$

Similarly, we can show that $\nu_{f(x)+A_{x}}(h(x)) \leq \nu_{g(x)+A_{x}}(h(x))$. Thus $g(x)+A_{x} \subseteq f(x)+A_{x}$. In a same way, we can show that $f(x)+A_{x} \subseteq g(x)+A_{x}$. Which complete the proof.

Theorem 3.18. Let $A$ be an IFI of $a$ ring $R$ and let $A_{x}$ be an IFPI of $R[x]$. Then

$$
R[x] / A_{x} \cong R[x] / A_{*}[x] .
$$

Proof. Define an map $\gamma: R[x] \rightarrow R[x] / A_{x}$ by $\gamma(f(x))=f(x)+A_{x}, \forall f(x) \in R[x]$.

Then it is easy to see that the map $\gamma$ is an epimorphism of rings with $\operatorname{Ker} \gamma$, where

$$
\begin{aligned}
\text { Ker } \gamma & =\left\{f(x) \in R[x]: \gamma(f(x))=A_{x}\right\} \\
& =\left\{f(x) \in R[x]: f(x)+A_{x}=A_{x}\right\} \\
& =\left\{f(x) \in R[x]: A_{x}(f(x)-0)=A_{x}(0)\right\} \\
& =\left\{f(x) \in R[x]: A_{x}(f(x))=A_{x}(0)\right\} \\
& =\left\{f(x) \in R[x]: \mu_{A_{x}}(f(x))=\mu_{A_{x}}(0) \text { and } \nu_{A_{x}}(f(x))=\nu_{A_{x}}(0)\right\} \\
& =\left\{f(x) \in R[x]: f(x) \in\left(A_{x}\right)_{*}\right\} \\
& =\left\{f(x) \in R[x]: f(x) \in A_{*}[x]\right\} \\
& =A_{*}[x] .
\end{aligned}
$$

The result follows by first theorem of homomorphism of rings.

\section{Prime and maximal intuitionistic fuzzy polynomial ideals}

In this section, we study some properties of the prime and maximal intuitionistic fuzzy polynomial ideals.

Definition 4.1. An intuitionistic fuzzy ideal $P$ of a ring $R$, not necessary constant, is said to be an intuitionistic fuzzy prime ideal, if for any IFIs $A$ and $B$ of $R$ the condition $A B \subseteq P$ implies that either $A \subseteq P$ or $B \subseteq P$.

Proposition 4.2. Let $A$ is an intuitionistic fuzzy prime ideal of a ring $R$, then $A_{*}$ is a prime ideal of $R$.

Proposition 4.3. Let $J$ be an ideal of a ring $R$ such that $J \neq R$. Then $J$ is a prime ideal of $R$ if and only if the IFS $A=\left(\mu_{A}, \nu_{A}\right)$ on $R$ defined by 
$\mu_{A}(x)=\left\{\begin{array}{ll}1, & \text { if } x \in J \\ \alpha, & \text { if otherwise }\end{array} ; \quad \nu_{A}(x)=\left\{\begin{array}{ll}0, & \text { if } x \in J \\ \beta, & \text { if otherwise }\end{array}, \alpha, \beta \in[0,1]\right.\right.$ such that $\alpha+\beta \leq 1$, is an intuitionistic fuzzy prime ideal of $R$.

Theorem 4.4. Let $A$ be an IFI of a ring $R$. Then $A$ is an intuitionistic fuzzy prime ideal of $R$ if and only if $A_{x}$ is an intuitionistic fuzzy prime ideal of $R[x]$.

Proof. Let $A$ be an intuitionistic fuzzy prime ideal of $R$, then $A_{*}$ is a prime ideal of $R$. By Theorem (3.4), $A_{x}$ is an intuitionistic fuzzy ideal of $R[x]$. To show that $A_{x}$ is an intuitionistic fuzzy prime ideal of $R[x]$, we have to show that, by Theorem (3.9), $\left(A_{x}\right)_{*}=A_{*}[x]$ is a prime ideal of a ring $R[x]$.

Assume that $A_{*}[x]$ is not a prime ideal of $R[x]$. Then there exists polynomials $f(x):=$ $\sum_{i=0}^{n} a_{i} x^{i}, g(x):=\sum_{i=0}^{m} b_{i} x^{i} \in R[x]$ such that $f(x) g(x) \in A_{*}[x]$, but $f(x), g(x) \notin A_{*}[x]$.

Let $i$ be the first smallest non-negative integer such that $\mu_{A}\left(a_{i}\right) \neq \mu_{A}(0)$ and $\nu_{A}\left(a_{i}\right) \neq \nu_{A}(0)$ and let $j$ be the first smallest non-negative integer such that $\mu_{A}\left(b_{i}\right) \neq \mu_{A}(0)$ and $\nu_{A}\left(b_{i}\right) \neq \nu_{A}(0)$. Since $f(x) g(x) \in A_{*}[x]$ implies that $\Sigma_{p, q=0, p+q=i+j}^{i+j} a_{p} b_{q} \in A_{*}$, since $a_{p}$ (where $p=0,1, \ldots, i-1$ ) and $b_{p}$ (where $p=0,1, \ldots, j-1$ ) are all in $A_{*}$, we have $a_{i} b_{j} \in A_{*}$. Since $A_{*}$ is prime ideal of $R$, either $\mu_{A}\left(a_{i}\right)=\mu_{A}(0)$ and $\nu_{A}\left(a_{i}\right)=\nu_{A}(0)$ or $\mu_{A}\left(b_{i}\right)=\mu_{A}(0)$ and $\nu_{A}\left(b_{i}\right)=\nu_{A}(0)$, a contradiction. Thus $A_{x}$ is an intuitionistic fuzzy prime ideal of $R[x]$.

Conversely, assume that $A_{x}$ is an intuitionistic fuzzy prime ideal of $R[x]$. We claim that $A_{x}$ is a prime ideal of $R$. Let $a, b \in R$ such that $a b \in A_{*}$. Then $(a x)(b x)=a b x^{2} \in A_{*}[x]=\left(A_{x}\right)_{*}$ . Since $\left(A_{x}\right)_{*}$ is a prime ideal of $R[x]$, either $(a x) \in\left(A_{x}\right)_{*}$ or $(b x) \in\left(A_{x}\right)_{*}$, which shows that either $a \in A_{*}$ or $b \in A_{*}$. This proves that $A$ is an intuitionistic fuzzy prime ideal of $R$.

Theorem 4.5. Let $f: R \rightarrow R^{\prime}$ be an epimorphism from $R$ onto $R^{\prime}$ and let $B$ be an intuitionistic fuzzy prime ideal of $R^{\prime}$ if and only if $f^{-1}(B)$ is an intuitionistic fuzzy prime ideal of $R$.

Proof. Firstly, assume that $B$ is an intuitionistic fuzzy prime ideal of $R^{\prime}$. Then $B_{*}$ is a prime ideal of $R^{\prime}$. Clearly, $f^{-1}(B)$ is an IFI of $R$. We claim that $\left(f^{-1}(B)\right)_{*}$ is a prime ideal of $R$. Let $a, b \in R$ be any element such that $a b \in\left(f^{-1}(B)\right)_{*}$. Then $\mu_{f^{-1}(B)}(a b)=\mu_{f^{-1}(B)}(0)$ and $\nu_{f^{-1}(B)}(a b)=\nu_{f^{-1}(B)}(0)$, i.e., $\mu_{B}(f(a b))=\mu_{B}\left(0^{\prime}\right)$ and $\nu_{B}(f(a b))=\nu_{B}\left(0^{\prime}\right) \Rightarrow f(a) f(b)=$ $f(a b) \in B_{*}$. Since $B_{*}$ is a prime ideal of $R^{\prime}$, either $f(a) \in B_{*}$ or $f(b) \in B_{*}$. Which means that either $\mu_{B}(f(a))=\mu_{B}\left(0^{\prime}\right)$ and $\nu_{B}(f(a))=\nu_{B}\left(0^{\prime}\right)$ or $\mu_{B}(f(b))=\mu_{B}\left(0^{\prime}\right)$ and $\nu_{B}(f(b))=$ $\nu_{B}\left(0^{\prime}\right)$, i.e., either $\mu_{f^{-1} B}(a)=\mu_{f^{-1} B}(0)$ and $\nu_{f^{-1} B}(a)=\nu_{f^{-1} B}(0)$ or $\mu_{f^{-1} B}(b)=\mu_{f^{-1} B}(0)$ and $\nu_{f^{-1} B}(b)=\nu_{f^{-1} B}(0)$, i.e., either $a \in\left(f^{-1}\right)(B)_{*}$ or $b \in\left(f^{-1}\right)(B)_{*}$.

Theorem 4.6. Let $f: R \rightarrow R^{\prime}$ be an epimorphism from $R$ onto $R^{\prime}$ and let $A$ be an $f$-invariant intuitionistic fuzzy ideal of $R$. Then $A$ is an intuitionistic fuzzy prime ideal of $R$ if and only if $f\left(A_{*}\right)$ is an intuitionistic fuzzy prime ideal of $R^{\prime}$.

Proof. Firstly, assume that $A$ is an intuitionistic fuzzy prime ideal of $R$. Then $A_{*}$ is a prime ideal of $R$. Let $x, y \in R^{\prime}$ such that $x y \in f\left(A_{*}\right)$. Since $f$ is onto, there exists $c \in A_{*}$ such that $f(c)=x y$ and there exists $a, b \in R$ such that $f(a)=x, f(b)=y$. Since $f(a b)=f(a) f(b)=x y=f(c)$. 
As $A$ is $f$-invariant, therefore, $\mu_{A}(a b)=\mu_{A}(c)=\mu_{A}(0)$ and $\nu_{A}(a b)=\nu_{A}(c)=\nu_{A}(0)$. Thus $a b \in A_{*}$. Since $A_{*}$ is a prime ideal of $R$, either $a \in A_{*}$ or $b \in A_{*}$, which shows that either $x=f(a) \in f\left(A_{*}\right)$ or $y=f(b) \in f\left(A_{*}\right)$. Hence $f\left(A_{*}\right)$ is a prime ideal of $R^{\prime}$.

Conversely assume that $f\left(A_{*}\right)$ is a prime ideal of $R^{\prime}$ and let $a, b \in R$ such that $a b \in A_{*}$. Thus $f(a) f(b)=f(a b) \in f\left(A_{*}\right)$. Since $f\left(A_{*}\right)$ is a prime ideal of $R^{\prime}$, either $f(a) \in f\left(A_{*}\right)$ or $f(b) \in f\left(A_{*}\right)$, which implies that either there exist $a^{\prime} \in A_{*}$ such that $f(a)=f\left(a^{\prime}\right)$ or there exist $b^{\prime} \in A_{*}$ such that $f(b)=f\left(b^{\prime}\right)$. Since $A$ is $f$-invariant, either $\mu_{A}(a)=\mu_{A}\left(a^{\prime}\right)=\mu_{A}(0)$ and $\nu_{A}(a)=\nu_{A}\left(a^{\prime}\right)=\nu_{A}(0)$ or $\mu_{A}(b)=\mu_{A}\left(b^{\prime}\right)=\mu_{A}(0)$ and $\nu_{A}(b)=\nu_{A}\left(b^{\prime}\right)=\nu_{A}(0)$, i.e., either $a \in A_{*}$ or $b \in A_{*}$. Hence $A_{*}$ is a prime ideal of $R$ and hence $A$ is an intuitionistic fuzzy prime ideal of $R$.

Corollary 4.7. Let $f: R \rightarrow R^{\prime}$ be an epimorphism from $R$ onto $R^{\prime}$ and let $A$ be an $f$-invariant intuitionistic fuzzy ideal of $R$. Then $A$ is an intuitionistic fuzzy prime ideal of $R$ if and only if $f(A)$ is an intuitionistic fuzzy prime ideal of $R^{\prime}$

Corollary 4.8. Let $f: R \rightarrow R^{\prime}$ be an epimorphism from $R$ onto $R^{\prime}, f_{x}$ be an induced homomorphism of $f$. Then an IFI $B$ of $R^{\prime}$ is an intuitionistic fuzzy prime ideal of $R^{\prime}$ if and only if $f_{x}^{-1}\left(B_{x}\right)$ is an intuitionistic fuzzy prime ideal of $R[x]$.

Corollary 4.9. Let $f: R \rightarrow R^{\prime}$ be an epimorphism from $R$ onto $R^{\prime}, f_{x}$ be an induced homomorphism of $f$. Then an IFI $A$ of $R$ is an intuitionistic fuzzy prime ideal of $R$ if and only if $f_{x}\left(A_{x}\right)$ is an intuitionistic fuzzy prime ideal of $R^{\prime}[x]$.

Definition 4.10. ([10]) A non-constant intuitionistic fuzzy ideal $A$ of a ring $R$ is called an intuitionistic fuzzy maximal ideal if for any intuitionistic fuzzy ideal $B$ of $R$, if $A \subseteq B$, then either $B_{*}=A_{*}$ or $B_{*}=R$.

Theorem 4.11. Let $A$ be a non-constant intuitionistic fuzzy ideal of a ring $R$. Then $A_{x}$ is maximal intuitionistic fuzzy ideal of $R[x]$, then $A$ is an intuitionistic fuzzy maximal ideal of $R$.

Proof. Let $A$ (non-constant) and $B$ be IFIs of a ring $R$ such that $A \subseteq B$ which implies $A_{x}$ and $B_{x}$ are IFIs of $R[x]$ such that $A x \subseteq B_{x}$. Now, $A_{x}$ is maximal intuitionistic fuzzy ideal of $R[x]$ then either $\left(B_{x}\right)_{*}=\left(A_{x}\right)_{*}$ or $\left(B_{x}\right)_{*}=R[x]$, i.e., either $B_{*}[x]=A_{*}[x]$ or $B_{*}[x]=R[x]$, i.e., either $B_{*}=A_{*}$ or $B_{*}=R$. Hence $A$ is an intuitionistic fuzzy maximal ideal of $R$.

Example 4.12. Let $\mathbf{Z}$ be the set of all integers. Define and IFS $A$ on $\mathbf{Z}$ by

$$
\mu_{A}(x)=\left\{\begin{array}{ll}
1, & \text { if } x \in 2 \mathbf{Z} \\
0, & \text { if otherwise }
\end{array} ; \quad \nu_{A}(x)= \begin{cases}0, & \text { if } x \in 2 \mathbf{Z} \\
1, & \text { if otherwise }\end{cases}\right.
$$

Then $A$ is an intuitionistic fuzzy maximal ideal of $\mathbf{Z}$, for if $B$ be any other IFI of $\mathbf{Z}$ such that $A \subseteq B$, then $B_{*}=A_{*}=2 \mathbf{Z}$ or $B_{*}=\mathbf{Z}$.

But $\left(A_{x}\right)_{*}=A_{*}[x]=\left\{f(x): f(x)=\sum_{i=0}^{n} a_{i} x^{i}, a_{i} \in A_{*}\right\}=\langle 2\rangle$ is not a maximal ideal of $\mathbf{Z}[x]$, since $\langle 2\rangle \subseteq\langle 2, x\rangle \subseteq \mathbf{Z}[x]$. Hence $A_{x}$ is not an intuitionistic fuzzy maximal ideal of $\mathbf{Z}[x]$. 


\section{Acknowledgements}

The second author would like to thank IKG PT University, Jalandhar for providing the opportunity to do research work.

\section{References}

[1] Atanassov, K. T. (1986) Intuitionistic fuzzy sets, Fuzzy Sets and Systems, 20(1), 87-96.

[2] Atanassov, K. T. (1999) Intuitionistic Fuzzy Sets: Theory and Applications, Studies on Fuzziness and Soft Computing, 35, Springer Physica-Verlag, Heidelberg.

[3] Bakhadach, I., Melliani, S., Oukessou, M., \& Chadli, S. L. (2016) Intuitionistic fuzzy ideal and intuitionistic fuzzy prime ideal in a ring, Notes on Intuitionistic Fuzzy Sets, 22(2), 5963.

[4] Banerjee, B., \& Basnet, D. K. (2003) Intuitionistic fuzzy subrings and ideals, The Journal of Fuzzy Mathematics, 11(1), 139-155.

[5] Biswas, R. (1989) Intuitionistic fuzzy subgroups, Math. Forum, 10, 37-46.

[6] Hur, K. , Kang, H. W., \& Song, H. K. (2003) Intuitionistic Fuzzy Subgroups and Subrings, Honam Math J., 25(1), 19-41.

[7] Hur, K., Jang, S. Y., \& Kang, H. W. (2005) Intuitionistic Fuzzy Ideals of a Ring, Journal of the Korea Society of Mathematical Education, Series B, 12(3), 193-209.

[8] Jun, Y. B., Ozturk, M. A., \& Park, C. H. (2007) Intuitionistic nil radicals of intuitionistic fuzzy ideals and Euclidean intuitionistic fuzzy ideals in ring, Information Science, 177, $4662-4677$.

[9] Kim, C. B., Kim, H. K., \& So, K. S. (2014) On the fuzzy polynomial ideals, Journal of Intelligent and Fuzzy Systems, 27, 487-494.

[10] Malik, D. S. (1991) Fuzzy Maximal, Radical, and Primary Ideals of a Ring, Information Sciences, 53, 237-250.

[11] Malik, D. S., \& Mordeson, J. N. (1998) Fuzzy Commutative Algebra, World Scientific Publishing Co-Pvt. Ltd.

[12] Meena, K. (2017) Characteristic intuitionistic fuzzy subrings of an intuitionistic fuzzy ring, Advances in Fuzzy Mathematics, 12(2), 229-253.

[13] Meena, K., \& Thomas, K. V. (2011) Intuitionistic L-Fuzzy Subrings, International Mathematical Forum, 12(52), 2561-2572.

[14] Sharma, P. K. (2011) Translates of intuitionistic fuzzy subring, International Review of Fuzzy Mathematics, 6(2), 77-84. 